\title{
Behavioral Alarm Treatment for Nocturnal Enuresis
}

\author{
Rodrigo F. Pereira, Edwiges F. M. Silvares, Paula F. Braga \\ Behavior Therapy Laboratory, Institute of Psychology, Universidade de Sao Paulo, Sao Paulo, SP, \\ Brazil
}

\begin{abstract}
Purposes: To investigate the efficacy of alarm treatment in a sample of Brazilian children and adolescents with nocturnal enuresis and relate treatment success to age and type of clinical support.

Materials and Methods: During 32 weeks, 84 children and adolescents received alarm treatment together with weekly psychological support sessions for individual families or groups of 5 to 10 families.

Results: $71 \%$ of the participants achieved success, defined as 14 consecutive dry nights. The result was similar for children and adolescents and for individual or group support. The time until success was shorter for participants missing fewer support sessions.

Conclusions: Alarm treatment was effective for the present sample, regardless of age or type of support. Missing a higher number of support sessions, which may reflect low motivation for treatment, increased the risk of failure.
\end{abstract}

Key words: enuresis; behavior therapy; child; adolescent; group therapy

Int Braz J Urol. 2010; 36: 332-8

\section{INTRODUCTION}

According to the International Children Continence Society, nocturnal enuresis is defined as discrete incontinence episodes while an individual is asleep (1). Children must be at least five years old to be diagnosed with enuresis. In addition to that, the DSM-IV criteria states that the involuntary voiding must occur at least twice a week for at least three months (2). Nocturnal enuresis is one of the most frequent problems of childhood, affecting up to $15 \%$ of children from 5 to 7 years of age and 1 to $2 \%$ of young adults (3-6). However, if DSM-IV criteria are employed, the prevalence of enuresis is around 2.6\% (3). The variation in the criteria employed by different investigators to define enuresis makes it difficult to establish a precise prevalence rate (5).

The etiology and underlying physiological mechanisms of nocturnal enuresis are heterogeneous
(7). Nevertheless, there is consensus concerning the notion that nocturnal enuresis arises from a combination of lack of vasopressin release during sleep or bladder hyperactivity and the inability to be aroused from sleep by bladder sensations (8). There clearly is a genetic basis at the origin of these phenomena (9), and the difficulty in waking up when the bladder is full is a sign of problems in the maturation of the central nervous system (10).

Both pharmacological and behavioral treatments are currently available for nocturnal enuresis. Desmopressin acetate, the most effective drug treatment, reduces the production of urine during the night, significantly decreasing wetting (11). The preferred behavioral treatment is alarm conditioning (12), associated with a success rate of $65 \%$ and $42 \%$ of relapse (13). Desmopressin acetate is no better than alarm or alarm plus desmopressin acetate in the long term (14). 
Some factors may affect the response to alarm treatment, especially those of a psychological nature, such as marital conflict, lack of motivation and parental punishment (13). The physiological aspects associated with a poor response to alarm treatment include the difficulty to wake up with the sound of the device (15). The literature concerning the impact of enuresis severity on the outcome of alarm treatment is contradictory, with both positive (13) and negative (16) impacts being reported.

There is a dearth of studies on the prevalence and severity of enuresis in the Brazilian population. A study carried out with a probabilistic sample in an urban center in southern Brazil (17) has revealed a 20.1\% prevalence of nocturnal enuresis in boys and $15.1 \%$ in girls based on the criterion of one wetting episode per night. This lack of studies may lead to a low level of information about enuresis and other lower urinary tract diseases among professionals that deal with children, such as caregivers and school teachers (18).

The main objective of the present study was to determine the success rate of alarm treatment in a population of Brazilian children and adolescents with nocturnal enuresis. We also aimed at identifying the relationship between age, type of psychological support and rate of success.

\section{MATERIALS AND METHODS}

The study sample included 84 children and adolescents from a university psychology clinic. Between 2002 and 2006, this group received care from four psychologists (graduate students at the university's Clinical Psychology graduate program). The participants were classified as children (6 to 10 years of age, $n=52$ ) or adolescents (11 to 17 years of age, $n=32$ ). Inclusion criteria were: age between 6 and 17 years, having wetting episodes at least twice a week for three consecutive months and absence of other disorders that could have caused the wetting episodes, such as diabetes or spina bifida. The sample included 19 adolescents that participated in the study conducted by Rocha, Costa and Silvares in the same research center (19).

All patients received full-spectrum home training (12), which consisted in the use of a bell- and-pad alarm during the night. Children and families were told to use the alarm daily in combination with cleanliness training and retention training as described by Houts (12). In addition, the families and patients were instructed to restrict fluid intake before going to bed, to keep regular sleep hours and to keep a record of night wetting episodes. Treatments lasted up to 32 weeks.

Each family participated in a weekly follow-up/support session lasting about one hour at the clinic. They were first screened for diagnosis and when there were about 20 families waiting for treatment, they were randomly assigned to participate in individual sessions $(\mathrm{n}=51)$ or group sessions including five to ten families $(\mathrm{n}=33)$. This procedure was undertaken about once or twice a year, and at each time a new randomization was made with the families previously screened. During the support sessions, the therapist reviewed the wetting record and made sure the procedures were being correctly followed. The children were accompanied by their parents or by caregivers in charge of monitoring the treatment at home.

Data were collected from the record filled out by the family, in which they informed whether the child was wet or dry on waking up. In the presence of bed wetting, the time when the alarm had rung and the approximate amount of urine, based on the size of wet patches (small, medium or large), were recorded. The treatment was considered to be successful if the child/adolescent remained dry for 14 consecutive nights during the treatment period. Treatment failure was defined as 13 or fewer consecutive dry nights or the family discontinuing the treatment (dropout). After success was achieved, a procedure (overlearning) to prevent relapse was performed. Overlearning involves drinking a small amount of water before going to sleep. The amount was determined according to the maximum voided volume expected for the child's age (age x $30+30 \mathrm{~mL}$ ) and was increased every two consecutive dry nights, until the child was able to remain dry another 14 nights after reaching initial success. The alarm treatment was interrupted when overlearning was complete or when it was attempted two times unsuccessfully. In these cases, after the second attempt, more 14 dry nights were required for finishing the program. 
Unpaired t-test was used to compare the frequency of enuresis episodes before treatment. Fisher's exact test was used to verify the relationship between success and the study variables (age and type of psychological support), and variance analysis was used to determine the time required to obtain success taking into consideration the study variables. ANOVA was used to analyze the variation in the time required to achieve success. Significance was established at $p$ $<0.05$.

\section{RESULTS}

To characterize the sample, the frequency of wetting episodes (severity of enuresis) in the sample was determined before the start of treatment. This information was not available for 10 participants, and thus 74 children and adolescents were considered $(\mathrm{N}=$ 74). Table- 1 shows the distribution of the sample according to the frequency of night wetting episodes.

Table-1 shows that more than half of the overall sample experienced bedwetting every night. This proportion was slightly larger in the group of adolescents, but the difference was not significant. Similarly, the mean number of weekly episodes for the overall sample (5.2) was similar to that for the two separate age groups.

The rate of severe enuresis in the sample is larger than that reported in prevalence studies (3). This is possibly due to the inclusion criteria used in this study and to the fact that the search for treatment may be more frequent when enuresis is more severe.
Table-2 shows the distribution of the two age groups and types of support in terms of treatment success and failure. Dropouts were included in the failure criteria.

Success was achieved in $71 \%$ of the sample. The success rate among children (6 to 10 years of age) and adolescents (11 to 17 years of age) was not statistically significant. Similarly, there was no statistical difference between the two types of support, although the failure rate was lower to participants receiving individual support ( $14 \%$ vs. $41 \%$ for group support) (Table-2). An analysis was also carried out to investigate if the onset of success differed in terms of age and session format. Figures 1 and 2 show the chance of obtaining success during treatment for these two variables.

Figure-1 shows that although success was achieved earlier in the group receiving individual support, the difference was not statistically significant. There was also no significant difference in time to achieve success according to age (Figure-2).

An analysis of time to achieve success related to session attendance is shown in Table-3.

The number of missed follow-up/support visits had a significant association with success: participants who missed less than $10 \%$ of sessions became dry faster than those who missed more than $10 \%$ of the sessions. It was also observed that the number of missed sessions was higher among those receiving group support: $62.5 \%$ of the participants missed more than $10 \%$ of the sessions vs. $37.5 \%$ of the participants receiving individual support $(\mathrm{p}=0.03)$.

Table 1 - Frequency of enuresis episodes before treatment.

\begin{tabular}{lcccc}
\hline & Overall & Children & Adolescents & p Value \\
\hline $\begin{array}{l}\text { Mean number of wet nights per week (SD) } \\
\text { Frequency of Bed Wetting Episodes per Week - \% (N) }\end{array}$ & $5.2(2.02)$ & $5.1(2.05)$ & $5.2(2.01)$ & 0.821 \\
2 to 3 & $17.6(13)$ & $17.1(7)$ & $18.2(6)$ & 0.818 \\
3 to 6 & $31.1(23)$ & $34.1(14)$ & $27.3(9)$ & \\
More than 6 & $51.4(38)$ & $48.8(20)$ & $54.5(18)$ \\
\hline
\end{tabular}


Table 2 - Success rate of an alarm technique with individual or group support in a sample of children and adolescents with nocturnal enuresis.

\begin{tabular}{|c|c|c|c|}
\hline & Success - N (\%) & Failure - N (\%) & p Value \\
\hline \multicolumn{4}{|c|}{ Overall } \\
\hline & $65(71)$ & $19(29)$ & Not applicable \\
\hline \multicolumn{4}{|c|}{ Age } \\
\hline Children & $41(79)$ & $11(21)$ & \multirow[b]{2}{*}{0.79} \\
\hline Adolescents & $24(75)$ & $8(25)$ & \\
\hline \multicolumn{4}{|c|}{ Type of Support } \\
\hline Group & $36(59)$ & $15(41)$ & \multirow{2}{*}{0.108} \\
\hline Individual & $29(86)$ & $4(14)$ & \\
\hline
\end{tabular}

\section{COMMENTS}

In the present study, the $71 \%$ success rate obtained by the participants is in accordance with previous results described in the literature - $65 \%$ on average (13). Considering more than half of our study participants had severe enuresis, the hypothesis that severity affects the results of treatment, either negatively or positively, may not be supported.

Age range has also been described as a predictor of failure, since adolescent enuresis is usually more difficult to treat (20). We did not observe a significant difference between children and adolescents, with both presenting similar success rates. However, with a broader sample, the slight difference in time to obtain success could appear as a significant factor.

The type of psychological support provided was not related to treatment success, as previously reported (13). Participants from families receiving individual support had similar success rates than those receiving group support; however, those receiving group support missed more sessions, an aspect associ- ated with greater difficulty in achieving success. The percentage of group format participants that missed more than $20 \%$ of the sessions was $39.6 \%$, against $17.5 \%$ of the participants of individual format. It is possible that the individual support leads to a greater commitment by the families, expressed in a better attendance to the sessions. Participants that missed more than $10 \%$ of the sessions, regardless of session format, took, on average, 5 more weeks to achieve success than those who missed less than $10 \%$ of the sessions. There seems to be a relationship between session attendance and success (13). The number of missed support sessions may be interpreted as a reflection of low adherence. It is likely that missing sessions in itself does not affect treatment, but rather, that it reflects a difficulty in following the prescribed steps, leading to a higher failure rate or to a longer interval until success achievement.

A limitation of this study was the absence of a control group and also of an analysis to determine the variability between therapists in terms of the support provided. In addition, a larger sample might

Table 3 - Time, in weeks, to achieve success (defined as 14 consecutive dry nights) according session attendance.

\begin{tabular}{lcccc}
\hline & & Mean & Standard Deviation & p Value \\
\hline Number of missed sessions & $<10 \%$ & 7.3 & 5.17 & $0.007^{*}$ \\
& $>10 \%$ & 13.3 & 8.20 & \\
\hline
\end{tabular}




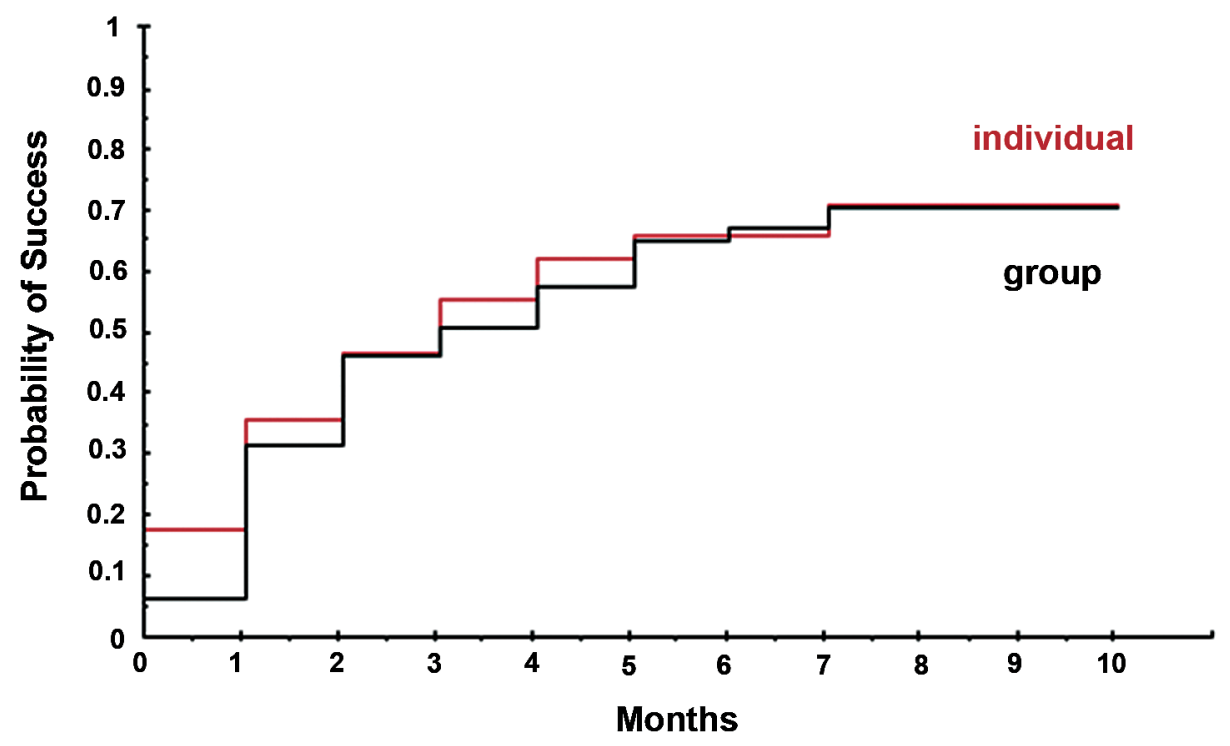

Figure 1 - Probability of success according to session format.

have been able to confirm the observed trend towards a better performance in participants receiving individual support, and to clarify the relationship between failure and missing support sessions. Therefore, it is not possible to determine which of these two juxtaposed variables determines treatment failure. A further limitation was the fact that treatment time was longer than that routinely practiced. This may

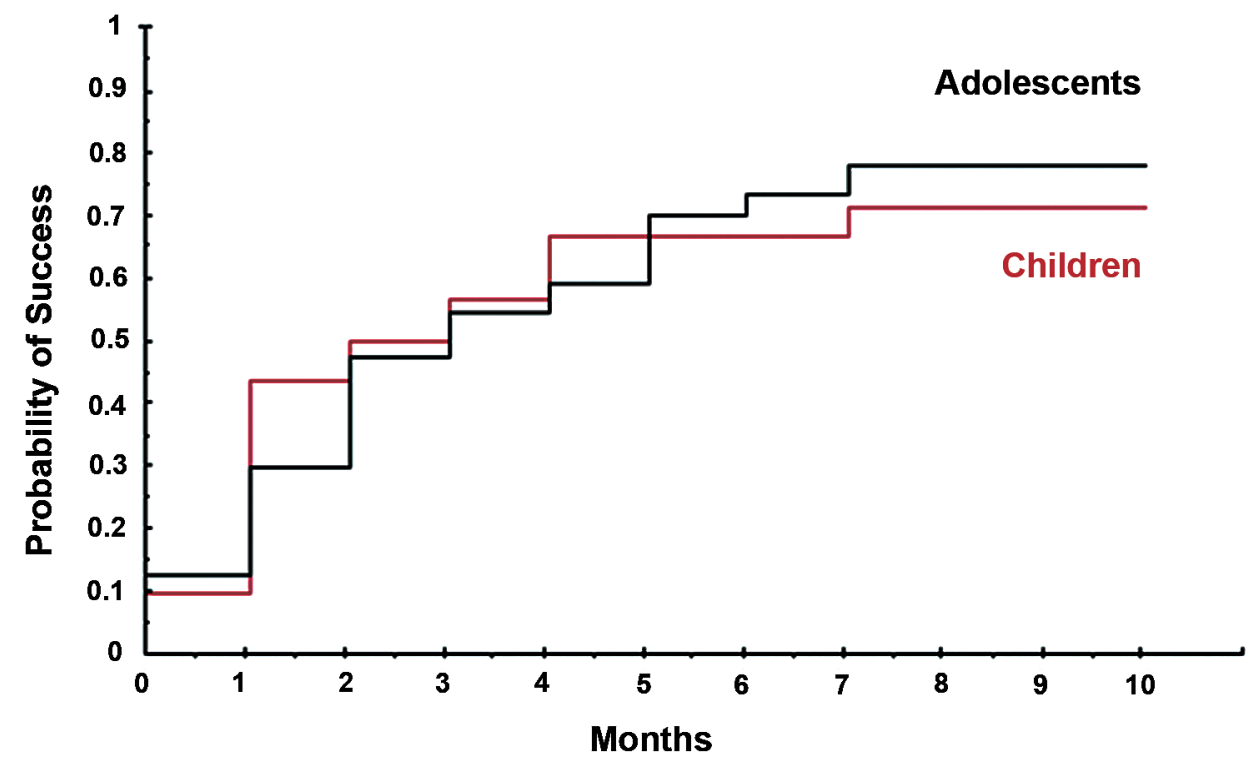

Figure 2 - Probability of success according to age. 
have exaggerated the success rate, since the cases of success may have resulted from spontaneous remission of enuresis.

\section{CONCLUSIONS}

Treatment of nocturnal enuresis with an alarm technique was satisfactory in this Brazilian sample. The success rate was in accordance with that described in the literature. The results of treatment were similar for children and adolescents and for individual and group support. Failure to participate in support sessions was associated with a delay in success achievement. We believe that treatment with the alarm technique may be used in other Brazilian patient populations and that additional studies should be carried out to identify predictors of success and failure that are characteristic of this population.

\section{ACKNOWLEDGEMENTS}

This work received financial support from Fundação de Amparo à Pesquisa do Estado de São Paulo (FAPESP) and Conselho Nacional de Desenvolvimento Científico e Tecnológico (CNPq).

\section{CONFLICT OF INTEREST}

None declared.

\section{REFERENCES}

1. Nevéus $T$, von Gontard $A$, Hoebeke $P$, Hjälmås $K$, Bauer S, Bower W, et al.: The standardization of terminology of lower urinary tract function in children and adolescents: report from the Standardisation Committee of the International Children's Continence Society. J Urol. 2006; 176: 314-24.

2. American Psychiatric Association: Diagnostic and Statistical Manual of Mental Disorders DSM-IV. Enuresis (Not Due to a General Medical Condition). Washington, DC. 1994; pp. 108-10.

3. Butler RJ, Golding J, Northstone K; ALSPAC Study Team: Nocturnal enuresis at 7.5 years old: prevalence and analysis of clinical signs. BJU Int. 2005; 96: 40410.

4. Lottmann H: Enuresis treatment in France. Scand J Urol Nephrol Suppl. 1999; 202: 66-9.

5. Butler RJ: Childhood nocturnal enuresis: developing a conceptual framework. Clin Psychol Rev. 2004; 24: 909-31.

6. Ozden C, Ozdal OL, Altinova S, Oguzulgen I, Urgancioglu G, Memis A: Prevalence and associated factors of enuresis in Turkish children. Int Braz J Urol. 2007; 33: 216-22.

7. Nevéus T: Diagnosis and management of nocturnal enuresis. Curr Opin Pediatr. 2009; 21: 199-202.

8. Hjalmas K, Arnold T, Bower W, Caione P, Chiozza LM, von Gontard A, et al.: Nocturnal enuresis: an international evidence based management strategy. J Urol. 2004; 171: 2545-61.

9. Järvelin MR, Vikeväinen-Tervonen L, Moilanen I, Huttunen NP: Enuresis in seven-year-old children. Acta Paediatr Scand. 1988; 77: 148-53.

10. Baeyens D, Roeyers H, Naert S, Hoebeke P, Vande Walle J: The impact of maturation of brainstem inhibition on enuresis: a startle eye blink modification study with 2-year followup. J Urol. 2007; 178: 2621-5.

11. Moffatt ME, Harlos S, Kirshen AJ, Burd L: Desmopressin acetate and nocturnal enuresis: how much do we know? Pediatrics. 1993; 92: 420-5.

12. Houts AC. Behavioral treatment for enuresis. In: Kazdin AE, Weisz JR, (ed.), Evidence-based psychotherapies for children and adolescents. New York, The Guilford Press. 2003; pp. 389-406.

13. Butler RJ, Gasson SL: Enuresis alarm treatment. Scand J Urol Nephrol. 2005; 39: 349-57.

14. Glazener CM, Evans JH, Peto RE: Alarm interventions for nocturnal enuresis in children. Cochrane Database Syst Rev. 2005; 18: CD002911.

15. Butler RJ, Robinson JC: Alarm treatment for childhood nocturnal enuresis: an investigation of withintreatment variables. Scand J Urol Nephrol. 2002; 36: 268-72.

16. Kristensen G, Jensen IN: Meta-analyses of results of alarm treatment for nocturnal enuresis--reporting practice, criteria and frequency of bedwetting. Scand J Urol Nephrol. 2003; 37: 232-8.

17. Mota DM, Victora CG, Hallal PC. Investigation of voiding dysfunction in a population-based sample of children aged 3 to 9 years. J Pediatr (Rio J). 2005; 81: 225-32.

18. Lordelo P, Maron F, Barros DG, Barroso DV, Bessa $\mathrm{J} \mathrm{Jr}$, Barroso U Jr: Lower urinary tract dysfunction in 
children. What do pre-school teachers know about it? Int Braz J Urol. 2007; 33: 383-8; discussion 388.

19. Rocha MM, Costa NJ, Silvares EF: Changes in parents' and self-reports of behavioral problems in Brazilian adolescents after behavioral treatment with urine alarm for nocturnal enuresis. Int Braz J Urol. 2008; 34: 74957; discussion 757.

20. Nappo S, Del Gado R, Chiozza ML, Biraghi M, Ferrara P, Caione P: Nocturnal enuresis in the adolescent: a neglected problem. BJU Int. 2002; 90: 912-7.

Accepted after revision:

January 18, 2010

\section{Correspondence address:}

Dr. Rodrigo Fernando Pereira

Av. Prof. Mello Moraes, 1721 / Bloco F / Sala 30

Cidade Universitária

São Paulo, SP, 05508-030, Brazil

E-mail: rpereira@usp.br

\section{EDITORIAL COMMENT}

The authors should be congratulated for performing a study evaluating the results with alarm for enuresis in Brazilian children and adolescents. The authors' good results with this treatment demonstrate their high level of expertise in this field and that Brazilian patients can be successfully treated by this method. However, a significant drawback of the alarm treatment is the high dropout rate (reference 13 and 14 in the article). An intention to treat analyses is the most accurate way to evaluate the final success rate and it was not the case in this study. For instance, the alarm was indicated for 100 children and 30 discontinued the treatment (dropouts) even before patients could be entered into the study protocol. Suppose 45 (65\%) out of those 70 had success with alarm. The rate of failure should be $55 \%(25+30$ patients failed $)$ not $35 \%$. Unfortunately, the overall dropout rate was not stated. How many patients were not included in the protocol because they or the family was not adapted to the method? Since we do not have this information, the results of this study can be interpreted as overestimated. Also, the lack of a control group, the limited number of patients and the absence of clear randomization criteria does not permit to draw any conclusions regarding the value of a psychological support for these types of patients. It is interesting to note that children have the same success rate as the adolescents showing that this treatment is successful even in younger age.

Dr. Ubirajara Barroso Jr. Rua Alameda dos Antúrios, 212 / 602 Salvador, BA, 40280-620, Brazil E-mail:ubarroso@uol.com.br 